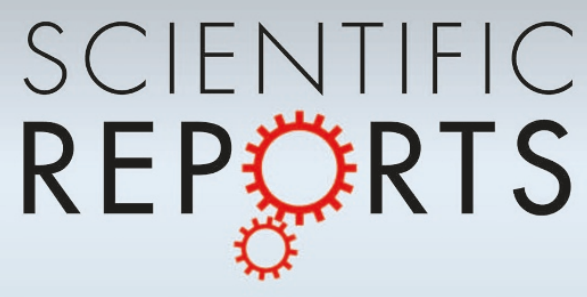

OPEN

SUBJECT AREAS:

CELL ADHESION

BIOPHYSICS

HAEMATOLOGICAL DISEASES

Received

15 August 2014

Accepted

2 March 2015

Published

31 March 2015

Correspondence and requests for materials should be addressed to

M.T. (tanaka@uniheidelberg.de) or A.D. H. (anthony_dick.ho@ urz.uni-heidelberg.de)

\section{Quantifying Adhesion Mechanisms and Dynamics of Human Hematopoietic Stem and Progenitor Cells}

\author{
Alexandra S. Burk ${ }^{1,2}$, Cornelia Monzel' , Hiroshi Y. Yoshikawa ${ }^{1,3}$, Patrick Wuchter ${ }^{4}$, Rainer Saffrich ${ }^{4}$, \\ Volker Eckstein ${ }^{4}$, Motomu Tanaka ${ }^{1,2,5}$ \& Anthony D. Ho ${ }^{4}$
}

${ }^{1}$ Physical Chemistry of Biosystems, Institute of Physical Chemistry, University of Heidelberg, 69120 Heidelberg, Germany, ${ }^{2}$ Institute of Toxicology and Genetics, Karlsruhe Institute of Technology, 76021 Karlsruhe, Germany, ${ }^{3}$ Department of Chemistry, Faculty of Science, Saitama University, Saitama, 338-8570, Japan, ${ }^{4}$ Department of Medicine V (Hematology, Oncology \& Rheumatology), University of Heidelberg, 69120 Heidelberg, Germany, ${ }^{5}$ Institute for Integrated Cell-Material Sciences (WPI iCeMS), Kyoto University, 606-8501, Kyoto, Japan.

Using planar lipid membranes with precisely defined concentrations of specific ligands, we have determined the binding strength between human hematopoietic stem cells (HSC) and the bone marrow niche. The relative significance of HSC adhesion to the surrogate niche models via SDF1a-CXCR4 or N-cadherin axes was quantified by (a) the fraction of adherent cells, (b) the area of tight adhesion, and (c) the critical pressure for cell detachment. We have demonstrated that the binding of HSC to the niche model is a cooperative process, and the adhesion mediated by the CXCR4- SDF1 $\alpha$ axis is stronger than that by homophilic $\mathrm{N}$-cadherin binding. The statistical image analysis of stochastic morphological dynamics unraveled that HSC dissipated energy by undergoing oscillatory deformation. The combination of an in vitro niche model and novel physical tools has enabled us to quantitatively determine the relative significance of binding mechanisms between normal HSC versus leukemia blasts to the bone marrow niche.

M ounting evidence has indicated that cellular and humoral determinants in the microenvironment play an essential role in governing the balance between self-renewal and differentiation of somatic stem cells. In the case of hematopoietic stem cells (HSC), adhesion to the niche in the bone marrow (BM) has been shown to maintain the dormancy of the most primitive HSC. The cellular determinants that might play a role include mesenchymal stromal cells (MSC), osteoblasts (OB), and vascular endothelial cells ${ }^{1-3}$. These cell types serve as surrogate niche to support HSC maintenance ${ }^{2,4,5}$. In the murine system, long-term HSC have been reported to adhere to $\mathrm{N}$-cadherin expressing, spindle-shaped osteoblasts ${ }^{1,6}$. It has been demonstrated that human $\mathrm{CD}_{3} 4^{+}$cells expressing N-cadherin ${ }^{7}$ are involved in early HSC differentiation ${ }^{8}$. In addition, overexpression of Ncadherin in HSC enhances adhesion and inhibits cell division of HSC in vitro, suggesting that N-cadherinmediated adhesion supports long-term maintenance of the HSC pool'. Mobilization and homing of HSC have been shown to be regulated by the sympathetic nervous system leading to secretion of stromal cell-derived factor $1 \alpha$ (SDF1 $\alpha$ ) by endothelial cells, OB and MSC ${ }^{10}$ and its interaction with the receptor CXCR4 expressed on HSC ${ }^{11}$. This multifunctional cytokine serves as chemoattractant for HSC and is suggested as the major player for HSC trafficking between the niche and peripheral blood ${ }^{12}$. Additionally, SDF1 $\alpha$ can be found as an immobilized molecule in the extracellular matrix of the endothelium ${ }^{13}$.

Acute myeloid leukemia (AML) is a clonal disorder of hematopoietic stem and progenitor cells that have lost the ability to differentiate into functional granulocytes or monocytes, hence leading to accumulation of undifferentiated leukemia blasts (LB) ${ }^{14}$. Present evidence indicates that the LB are derived from leukemia initiating cells (LIC). The latter are well protected from cell division by adhesion to the marrow niche, hence are resistant to chemotherapy and responsible for recurrence of disease ${ }^{15,16}$. Although HSC and LIC both reside in the microenvironment of the $\mathrm{BM}^{17,18}$, little is known about the precise molecular mechanisms and the differences in the interactions between marrow niche and HSC on the one hand, and LIC on the other. The differential adhesion of HSC versus LIC to the marrow niche might be exploited for the mobilization of LIC before chemotherapy. To quantitatively define the adhesive strength mediated by specific adhesion molecules binding human HSC to the niche, and to discriminate the potential differences as compared to their malignant counterparts, it is essential to 


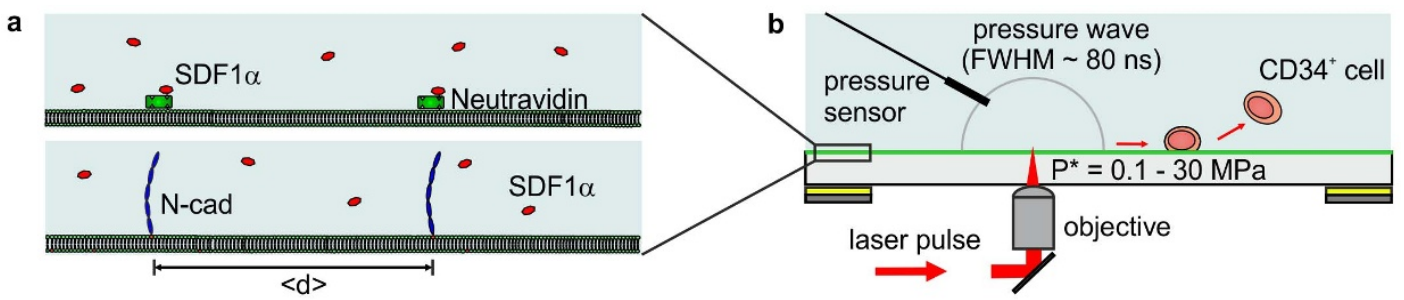

Figure 1 Experimental set-up. Schematic illustrations of (a) the defined surrogate MSC model based on supported membranes and (b) a novel assay to quantify the cell detachment pressure using a laser-induced pressure wave.

design highly controllable in vitro model systems that are based on studies on cellular determinants derived from human origin.

In the present study, we have designed a model of surrogate MSC surfaces by the deposition of planar lipid membranes (supported membranes) ${ }^{19,20}$ displaying specific ligands, such as $\mathrm{N}$-cadherin and SDF $1 \alpha$ (Fig. 1a). In contrast to commonly used assays that rely on counting the number of adherent HSC on a MSC feeder layer ${ }^{2}$, lipid membranes allow for the precise control of the average lateral distance between ligand molecules, e.g. specific proteins, to nanometer ( $\mathrm{nm}$ ) accuracy. In addition to label-free live cell image analysis with reflection interference contrast microscopy $(\mathrm{RICM})^{21}$, we employed a novel assay utilizing intensive pressure waves induced by laser pulses (Fig. 1b) to quantify the adhesion strength of HSC to the in vitro model niche ${ }^{22}$. This technique utilizes a "shock wave" (a pressure wave traveling at a speed beyond the sound velocity) that is induced by a picosecond (ps) laser pulse focused near the substrate surface. The reachable force exerted on a cell $(>1 \mathrm{mN})$ by such pressure waves could be more than six orders of magnitude larger than the typical force range achieved with optical traps ${ }^{23}$ or magnetic tweezers ${ }^{24}$. Such pressure waves are strong enough to detach cells from adhesive surfaces in a non-invasive manner. In contrast to alternative approaches such as peeling off a cell using an AFM tip $^{25}$, this novel assay guarantees statistically reliable data points, i.e. 10-20 cells within $20 \mathrm{~min}$. In addition, we analyzed the shape fluctuation of HSC by calculating the autocorrelation maps and corresponding power spectra in Fourier space in order to extract characteristic spatio-temporal patterns from the morphological dynamics of HSC in response to the in vitro model niche ${ }^{26,27}$. The use of statistical physics methods has enabled us to identify different modes of shape deformation and motion of HSC as well as to assess the energy dissipation by HSC in the presence and absence of SDF $1 \alpha$, which is usually hidden behind stochastic noises.

\section{Results}

Quantifying the relative significance of SDF1 $\alpha$ and N-cadherin. Figure $2 \mathrm{a}$ and $2 \mathrm{~b}$ represent the phase contrast images of HSC on supported membranes that displayed SDF1 $\alpha$ and $\mathrm{N}$-cadherin at

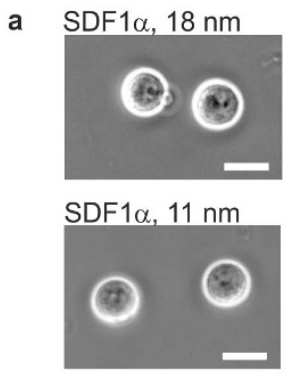

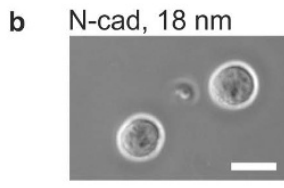

$\mathrm{N}-\mathrm{cad}, 11 \mathrm{~nm}$

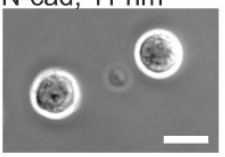

$<d>=18 \mathrm{~nm}$ (top) and $11 \mathrm{~nm}$ (bottom), respectively. The fraction of adherent HSC normalized by cells seeded on surrogate niche models was determined by counting the surface densities of adherent cells. As summarized in Fig. 2c (red), an increase in the average lateral distance $\langle d>$ of $\operatorname{SDF} 1 \alpha$ resulted in a significant decrease in the fraction of adherent HSC $\chi$, suggesting a distinct transition from the "bound" state (close to 100\%) to "unbound" state (close to the base line). This transition in cell adhesion can be interpreted with the empirical Hill equation ${ }^{28}$ :

$$
\chi=\chi_{\min }+\frac{\chi_{\max }-\chi_{\min }}{1-\left(d / d^{h a l f}\right)^{n}}
$$

where $\chi_{\min }$ and $\chi_{\max }$ stand for the minimum and maximum levels. To exclude any non-specific binding of HSC to the in vitro model niche, cells were seeded on a pure phospholipid membrane with no ligand molecules (Fig. 2c, black) as controls. No signs of adhesion was observed on the control surfaces.

The unbinding transition could be characterised by the critical distance $\left\langle d_{\mathrm{SDF} 1 \alpha}{ }^{*}\right\rangle \sim 27 \mathrm{~nm}$ and the cooperativity coefficient $n$ $\sim 3$, respectively. In contrast, the corresponding results for $\mathrm{N}$ cadherin (blue) exhibited a broader tail to a large intermolecular distance $\left\langle d_{\mathrm{N} \text {-cadherin }} *>\sim 50 \mathrm{~nm}\right.$ with a cooperativity coefficient $n \sim 2$, which was consistent with our previous reports on intercellular junctions between HSC and MSC via N-cadherin ${ }^{7,29}$. The results suggested that HSC specifically detected slight changes in $\langle d\rangle$ with $n m$ accuracy, and the adhesion mediated by both SDF1 $\alpha$-CXCR 4 and homophilic N-cadherin axes could be classified as cooperative reactions. Nevertheless, the determination of the fraction of adherent cells by such an "ensemble counting" does not allow for a clear discrimintation between strongly and weakly adherent cells. In order to gain a deeper insight into the cell adhesion behavior, we have calculated the area of tight adhesion $A_{a d h}$ from individual cells. Figures $3 \mathrm{a}$ and $3 \mathrm{~b}$ represent the Reflection Interference Contrast Microscopy (RICM) images of HSC on supported membranes displaying SDF1 $\alpha$ and $\mathrm{N}$-cadherin at $\langle d\rangle=18 \mathrm{~nm}$ and $11 \mathrm{~nm}$, respectively. Thus, RICM permitted the quantitative identification of minor changes in

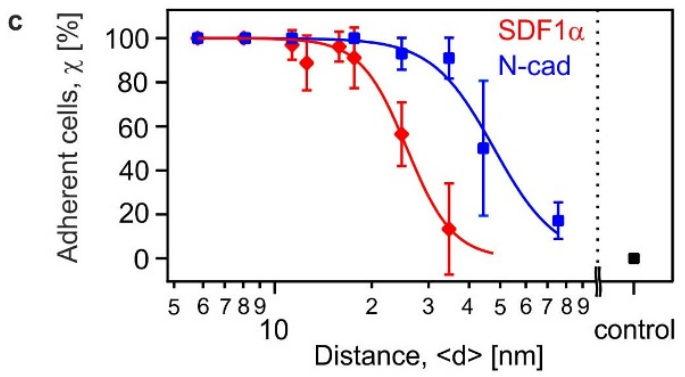

Figure $2 \mid$ HSC specifically detect changes in intermolecular ligand distance with $\mathbf{n m}$ accuracy. Phase contrast images of HSC on membranes displaying (a) SDF $1 \alpha$ at $\langle d\rangle=18 \mathrm{~nm}$ (top) and $\langle d\rangle=11 \mathrm{~nm}$ (bottom) and (b) N-cadherin at $\langle d\rangle=18 \mathrm{~nm}$ (top) and $\langle d\rangle=11 \mathrm{~nm}$ (bottom) at $t=2 \mathrm{~h}$ (scale bar: $10 \mu \mathrm{m}$ ). (c) The fraction of adherent HSC on supported membranes, plotted as functions of $\left\langle d_{\mathrm{N} \text {-Cadherin }}\right\rangle$ (blue) and $\left\langle d_{\mathrm{SDF} 1 \alpha}\right\rangle$ (red) could be characterised by an empirical Hill equation (solid lines), identifying a difference in the transition point. Data points represent means \pm SD for $\mathrm{n}=50$ cells. 


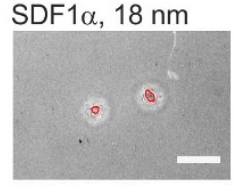

$\mathrm{SDF} 1 \alpha, 11 \mathrm{~nm}$

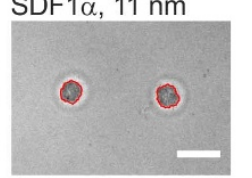

b

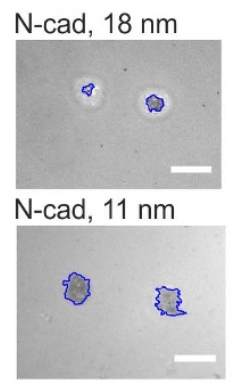

c

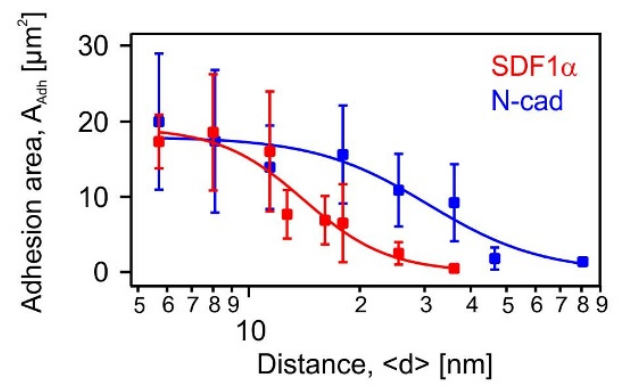

Figure $3 \mid$ The area of tight adhesion revealed a significant difference between N-cadherin and SDF1 $\alpha$ mediated binding. Micro-interferometry images of HSC on membranes displaying (a) SDF1 $\alpha$ at $\langle d\rangle=18 \mathrm{~nm}$ (top) and $\langle d\rangle=11 \mathrm{~nm}$ (bottom) and (b) N-cadherin at $\langle d\rangle=18 \mathrm{~nm}$ (top) and $\langle d\rangle$ $=11 \mathrm{~nm}$ (bottom) at $t=2 \mathrm{~h}$ (scale bar: $10 \mu \mathrm{m}$ ). (c) The average area of tight adhesion per cell determined by micro-interferometry, plotted versus $\left\langle d_{\mathrm{N} \text {-Cadherin }}\right\rangle$ (blue) and $\left\langle d_{\mathrm{SDF} 1 \alpha}\right\rangle$ (red) could be characterised by an empirical Hill equation (solid lines) by replacing $\chi$ with $A$. Data points represent means $\pm \mathrm{SD}$ for $\mathrm{n}=50$ cells.

$A_{A d h}$ in relationship to the different average lateral distances $\langle d\rangle$ between ligand molecules. Fig. $3 c$ represents the calculated area of tight adhesion per cell plotted as a function of $\left\langle d_{\mathrm{SDF} 1 \alpha}\right\rangle$ (red) and $<d_{\mathrm{N} \text {-cadherin }}>$ (blue). The area of tight adhesion exhibited a significant decrease from $16 \mu \mathrm{m}^{2}$ to $7 \mu \mathrm{m}^{2}$ when the average lateral distance increases from $\left\langle d_{\mathrm{SDF} 1 \alpha}\right\rangle=11 \mathrm{~nm}$ to $18 \mathrm{~nm}$. No adhesion zone could be detected at $\left\langle d_{\mathrm{SDF} 1 \alpha}\right\rangle \geq 34 \mathrm{~nm}$. The corresponding results on membranes displaying $\mathrm{N}$-cadherin also exhibited a similar tendency, whereas no signs of adhesion could be detected at $<d_{\mathrm{N} \text {-cadherin }}>>80 \mathrm{~nm}$. The clear discrimination in the area of tight adhesion at $\left\langle d_{\mathrm{SDF} 1 \alpha}\right\rangle \leq 18 \mathrm{~nm}$ presented in Fig. $3 \mathrm{c}$ was in contrast to the ensemble counting results in Fig. $2 c$, where the cells were merely classified either as "adherent" or "non-adherent". This finding implied that the RICM analysis was much more sensitive for assessment of the difference in cell adhesion. In fact, this might account for the apparent differences in the unbinding transition to a smaller critical distance, $\left\langle d^{*}\right\rangle=14 \mathrm{~nm}$ for SDF1 $\alpha$ and $31 \mathrm{~nm}$ for $\mathrm{N}$ cadherin, obtained by replacing $\chi$ to $A$ in equation 1 . Moreover, the cooperativity coefficients of SDF $1 \alpha$-CXCR 4 and $N$-cadherin axes were $n=1.9$ and 1.4 , respectively, verifying the cooperativity of both interactions. Although an active response of the cell via up- or downregulation of receptors might affect adhesion on longer time scale, no time-dependent change in the adhesion area $A_{a d h}$ could be detected for HSC on membranes displaying either SDF1 $\alpha$ or N-cadherin during a total observation time of $4 \mathrm{~h}$ (data not shown).

To quantify the mechanical strength of the binding between HSC and the in vitro niche model mediated by specific receptor/ligand pairs, we have utilized a novel technique based on cell detachment by pressure waves induced by ultra-short laser pulses ${ }^{22}$. In Figure 4a, single HSC are shown before and after their successful detachment demonstrating that cells were viable and adhered again to the in vitro niche model. Figure $4 \mathrm{~b}$ represents the critical pressures $P^{*}$ for the detachment of HSC plotted as a function of $\left\langle d_{\mathrm{N} \text {-cadherin }}\right\rangle$ and $\left\langle d_{\mathrm{SDF} 1 \alpha}\right\rangle$. The measurements were performed within the range of $\langle d\rangle=5.5-25 \mathrm{~nm}$, at which the majority of cells were identified as "adherent" (Fig. 2c). The critical pressure $P^{*}$ showed a clear decrease corresponding to the increase in $\langle d\rangle$ within this range, sharing common features with changes in the area of tight adhesion (Fig. 3c). It should be noted that these mechanical assays magnified differences in the strength of HSC binding on the niche model displaying SDF $1 \alpha$ and $\mathrm{N}$-cadherin at $\langle d\rangle$, which could not be identified from the area of tight adhesion (Fig. 3c). For example, the critical pressure $P^{*}$ to detach HSC from the niche model functionalized with SDF1 $\alpha$ at $\langle d\rangle=11 \mathrm{~nm}\left(P^{*}=6.4 \mathrm{MPa}\right)$ was more than two times higher than the corresponding value on membranes displaying $\mathrm{N}$-cadherin at the same average lateral distance $(2.8 \mathrm{MPa})$. Note that the direct comparison between the $P^{*}$ values obtained here and those obtained by other experiments (e.g. AFM, micropipette aspiration) is not possible due to the fact that the characteristic time window for the bond rupture is different by several orders of magnitude. As demonstrated by Merkel et $a .^{30}$, the rupture force for individual bonds is strongly dependent on the loading rate. In our experiments, the cells are exposed to the pressure wave only for $\sim 80 \mathrm{~ns}$, which is more than $10^{7}$ times shorter than the characteristic time windows for the other assays $\mathrm{s}^{25,31,32}$. In fact, such a short exposure to pressure waves eliminates both inelastic deformation of cells and remodeling of cytoskeleton during the cell detachment from our assays. It is also known that the ligand-receptor binding leads to force generation by the adherent cell towards the substrate. However, such mechanotransduction does not play a role in the case of SDF $1 \alpha$-CXCR4 binding, since SDF1 $\alpha$ binds to the extracellular domain of the receptor CXCR4 whose intracellular domain is associated with heterotrimeric $\mathrm{G}$ proteins ${ }^{33}$ and thus this process does not

a Before detachment
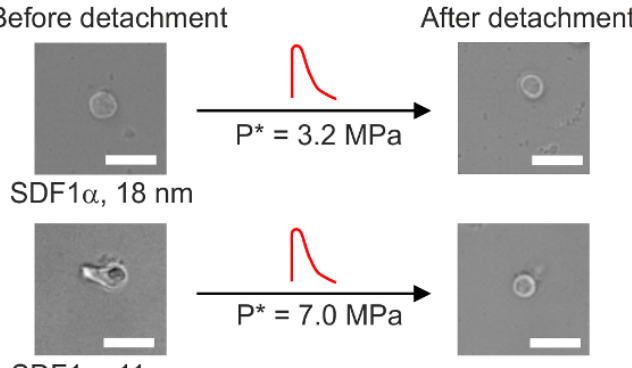

$$
\mathrm{SDF} 1 \alpha, 11 \mathrm{~nm}
$$



Figure $4 \mid$ SDF1 $\alpha$ mediates a higher adhesion strength compared to $\mathrm{N}$-cadherin. (a) Bright-field images of individual HSC on membranes displaying SDF1 $\alpha$ at $\langle d\rangle \sim 18 \mathrm{~nm}$ (upper) and $\langle d\rangle \sim 11 \mathrm{~nm}$ (lower) before and after its detachment by pressure waves (red waves) at $t=2 \mathrm{~h}$ (scale bar: $10 \mu \mathrm{m}$ ). Applying a laser-induced pressure wave, HSC were displaced from the substrate and shifted by about $10 \mu \mathrm{m}$. Subsequently, HSC were able to adhere again to the in vitro model niche as evidence for its viability. (b) Critical detachment pressures $P^{*}$ determined for HSC on supported membranes at $t=2 \mathrm{~h}$, plotted as functions of $\left\langle d_{\mathrm{N} \text {-cadherin }}\right\rangle$ (blue) and $\left\langle d_{\mathrm{SDF} 1 \alpha}\right\rangle$ (red). Data points represent means \pm SD for $\mathrm{n}=50$ cells. 
Table 1 | SDF $1 \alpha$ shifts the dynamic equilibrium between bound and unbound states of CXCR4-SDF $1 \alpha$. The fraction of adherent HSC $\alpha$, the average area of adhesion $A_{A d h}$, and the critical detachment pressure $P^{*}$ in the presence and absence of $5 \mathrm{ng} / \mathrm{mL} \mathrm{SDF} 1 \alpha$ at $t=2 \mathrm{~h}$. The experiments were performed at two different average lateral distances of $S D F 1 \alpha,<d_{S D F 1 \alpha}>=11 \mathrm{~nm}$ and $18 \mathrm{~nm}$. The values represent means \pm SD for $n=50$ cells

\begin{tabular}{|c|c|c|c|c|c|c|}
\hline \multirow[b]{2}{*}{$<\mathrm{d}>[\mathrm{nm}]$} & \multicolumn{2}{|c|}{$\chi[\%]$} & \multicolumn{2}{|c|}{$\mathrm{A}_{\text {Adh }}\left[\mu \mathrm{m}^{2}\right]$} & \multicolumn{2}{|c|}{$\mathrm{P} *[\mathrm{MPa}]$} \\
\hline & 11 & 18 & 11 & 18 & 11 & 18 \\
\hline $\begin{array}{l}\text { without SDF } 1 \alpha \\
\text { with SDF } 1 \alpha\end{array}$ & $\begin{array}{l}97.0 \pm 6.7 \\
93.1 \pm 6.7\end{array}$ & $\begin{array}{l}94.0 \pm 14.1 \\
52.0 \pm 24.4\end{array}$ & $\begin{array}{l}16.0 \pm 8.0 \\
9.5 \pm 6.4\end{array}$ & $\begin{array}{l}6.5 \pm 2.0 \\
2.0 \pm 1.9\end{array}$ & $\begin{array}{l}7.0 \pm 2.5 \\
3.6 \pm 0.7\end{array}$ & $\begin{array}{l}3.2 \pm 0.2 \\
2.2 \pm 0.4\end{array}$ \\
\hline
\end{tabular}

involve any direct participation of the cytoskeleton. In the case of homophilic N-cadherin binding, it has been reported that the cell adhesion on substrates displaying immobilized N-cadherin leads to the formation of adherens junctions $s^{34}$. Although the extracellular domains of $\mathrm{N}$-cadherin anchored on supported membranes via lipid anchors can undergo lateral diffusion on membrane surfaces, we observed no distinct change in the shape or area of the tight adhesion within our experimental time window (up to $4 \mathrm{~h}$ after seeding).

Influence of soluble SDF1 $\alpha$ on adhesion. Since SDF1 $\alpha$ is not only expressed by MSC but also released in the extracellular space of the $\mathrm{BM}$ microenvironment ${ }^{10}$, the fraction of adherent HSC, the area of tight adhesion, and the critical pressure for cell detachment pressure were measured in the presence or absence (as control) of $5 \mathrm{ng} / \mathrm{mL}$ SDF $1 \alpha$, which corresponded to the physiological level of SDF1 $\alpha$ in human BM. We have identified a decrease in the adhesion strength by a distinct decrease in the adhesion area and the critical pressure for cell detachment at $t=2 \mathrm{~h}$ in the presence of soluble SDF1 $\alpha$ (Table 1). For example, at $\left\langle d_{\mathrm{SDF} 1 \alpha}\right\rangle=11 \mathrm{~nm}$, the area of tight adhesion and the critical detachment pressure were reduced almost by a factor of two in the presence of soluble SDF1 $\alpha$. Our finding suggested that the presence of soluble SDF1 $\alpha$ was able to shift the dynamic equilibrium between bound and unbound states of CXCR4-SDF1 $\alpha^{35}$. The decrease in the HSC adhesion area was much more pronounced compared to that observed in previous experiments on substrates coated with fibronectin, where no significant difference in cell adhesion could be observed until soluble SDF1 $\alpha$ was applied at concentrations $s^{36,37}$. The SDF1 $\alpha$ concentrations used in these studies were 1-2 orders of magnitude higher than the physiological concentration we used in this study $(5 \mathrm{ng} / \mathrm{mL})$. However, as these studies investigated MSC-HSC interactions and thus cannot disriminate different ligand-receptor pairs, the direct comparison with our results is practically invalid. To define whether soluble SDF1 $\alpha$ affects the HSC adhesion mediated by the homophilic Ncadherin axis, we also examined HSC on supported membranes functionalized with $\mathrm{N}$-cadherin in the presence and absence of soluble SDF1 $\alpha(5 \mathrm{ng} / \mathrm{mL})$ at $\left\langle d_{N-c a d}\right\rangle \sim 18-47 \mathrm{~nm}$. The results clearly showed that soluble SDF1 $\alpha$ did not affect the fraction of adherent HSC, the average area of tight adhesion, nor on the critical detachment pressure $P^{*}$ mediated by N-cadherin (see Supplementary Table S1).

Correlation between morphological dynamics and modes of motion. We have then addressed the issue how the HSC-niche interaction is reflected on the morphological dynamics and the mode of locomotion. Such analyses have been applied extensively to characterize the mainly thermal fluctuations in model membranes and erythrocytes ${ }^{38-40}$. In case of nucleated cells the dynamic fluctuations comprise mostly active processes such as the bending and stretching of cellular membranes, rearrangements of molecules embedded therein or the remodeling of cytoskeletal structures ${ }^{39,41}$. Thus, the power spectrum analyses yield additional insight into the energy dissipation through cellular deformation. As previously reported, SDF1 $\alpha$ is not only a chemoattractant to HSC but also induces cytoskeleton remodeling and thus changes in HSC morphology ${ }^{37}$. To extract characteristic spatio-temporal patterns from stochastic dyamics of HSC, the peripheral edge of the cells was defined from the phase contrast time-lapse images using the contrast in the pixel values (see Supplementary Fig. 2). In the next step, the radial distance $r$ between the edge of the cell and the center of mass was plotted as a function of angle $\theta=0-180^{\circ}$ over time $t$, $r(\theta, t)$ yielding the morphological dynamics of the target cell. The characteristic spatio-temporal patterns were extracted by calculating the autocorrelation function $\Gamma_{\mathrm{rr}}(\theta, t)$ of the stochastic amplitude map $r(\theta, t)$ :

$$
\Gamma_{r r}(\theta, t)=\frac{\langle r(\theta+\Delta \theta, t+\Delta t) \cdot r(\theta, t)\rangle}{\left\langle[r(\theta, t)]^{2}\right\rangle}
$$

As presented in Fig. 5, using the phase contrast images and the trajectory of HSC tracked over 90 min $(\mathrm{a}-\mathrm{c})$, the amplitude maps (df) and the autocorrelation maps ( $\mathrm{g}-\mathrm{i})$, surrogate parameters for cellular locomotion could be subdivided into three major categories: (1) At low average lateral distance of $S D F 1 \alpha$, e.g. $\left\langle d_{\mathrm{SDF} 1 \alpha}\right\rangle=6 \mathrm{~nm}$, the projected HSC morphology remained round and could be characterised by a featureless amplitude map (Fig. 5d). Furthermore, a quick decay of autocorrelation over time (Fig. $5 \mathrm{~g}$ ) suggested that HSC underwent a rotational motion. This finding implied that HSC had little degree of freedom to deform its periphery on sticky surfaces with low average lateral distances $\langle d\rangle$ of adhesive ligands and the translational motion was blocked. (2) At a lateral intermolecular distance $\left\langle d_{\mathrm{SDF} 1 \alpha}\right\rangle=11$ and $18 \mathrm{~nm}$, HSC extended a

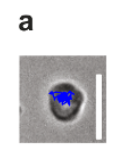

b
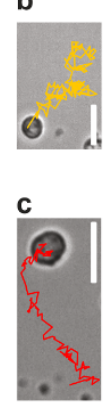

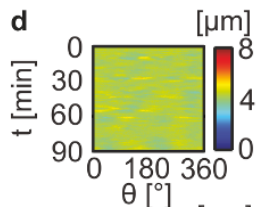

e

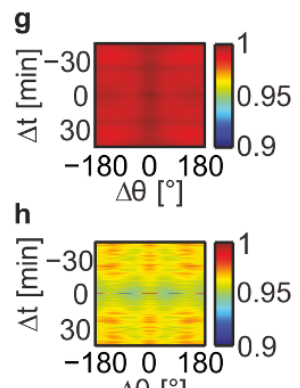

$\Delta \theta\left[^{\circ}\right]$

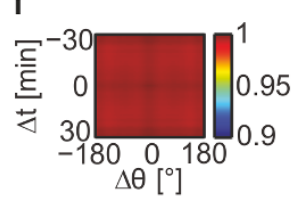

Figure 5 | HSC motion can be categorized into three major classes. Morphological dynamics of HSC on supported membranes displaying SDF1 $\alpha$ : $(\mathrm{a}-\mathrm{c})$ phase contrast images and trajectories over $90 \mathrm{~min},(\mathrm{~d}-\mathrm{f})$ amplitude $r(\theta, t)$ maps, and $(\mathrm{g}-\mathrm{i})$ the corresponding autocorrelation $\Gamma_{\mathrm{r}, \mathrm{r}}(\theta, t)$ maps. The patterns could be classified into three representative cases as a function of $\left\langle d_{\mathrm{SDF} 1 \alpha}>; 6 \mathrm{~nm}(\mathrm{a}, \mathrm{d}, \mathrm{g}), 18 \mathrm{~nm}(\mathrm{~b}, \mathrm{e}, \mathrm{h})\right.$ and $34 \mathrm{~nm}$ $(\mathrm{c}, \mathrm{f}, \mathrm{i})$. (scale bars: $10 \mu \mathrm{m})$. 
pseudopod, followed by an extension in a perpendicular direction (Fig. 5b, e, h). Such an oscillatory deformation could be identified from the periodic appearance of peaks at $\theta=0 / 180^{\circ}$ and $90 / 270^{\circ}$ in the autocorrelation map (Fig. 5h). This finding suggested that HSC first stretched the pseudopods along the long axis to find the binding spots, then retracted to a round shape, and extended the pseudopods in a perpendicular direction. (3) When the average lateral distance further increased, e.g. $\left\langle d_{\mathrm{SDF} 1 \alpha}\right\rangle=34 \mathrm{~nm}$, the deformation caused by cell adhesion became less prominent during the translational displacement (Fig. 5f, i).

Cell deformation, such as bending of cell membranes and remodeling of cytoskeletons ${ }^{39,40,41}$, is an active processes that consumes energy. Here, we performed the mode analysis of the power spectrum from the Fourier transform of amplitude maps $F T(r(\theta, t))$ for $\mathrm{m}=0$, $1,2,3, \ldots$ in a similar way to Partin et al. ${ }^{42}$ but calculated in the inertial frame with the origin being the center of mass (i.e. the translational mode was not assessed). This enabled one to quantitatively determine the predominant mode of deformation that HSC consumes the energy:

$$
\hat{\Gamma}_{r, r}(\theta, t)=\langle F T(r(\theta, t)) * F T(r(\theta, t))\rangle
$$

The calculated power spectrum of HSC at various $\left\langle d_{\mathrm{SDF} 1 \alpha}\right\rangle$ values exhibited peaks at mode number $\mathrm{m}=2$, implying that the mode of HSC deformation was predominantly oscillatory (inset) independent from $\left\langle d_{\mathrm{SDF} 1 \alpha}\right\rangle$ (Fig. 6a). In contrast, the deformation at mode $\mathrm{m}=1$ was suppressed. Moreover, looking at the power spectra calculated for different average lateral distances between SDF1 $\alpha<d_{\mathrm{SDF} 1 \alpha}>$ (indicated by different colors in Fig. $6 \mathrm{a}$ ), the amount of energy dissipated by the cell through shape deformation did not monotonically follow the changes in the average lateral distance $\langle d\rangle$, reaching the maximum at around $\left\langle d_{\mathrm{SDF} 1 \alpha}\right\rangle=11$ and $18 \mathrm{~nm}$. This finding suggests that the magnitude of energy consumed by the deformation of HSC is not only determined by the intermolecular distance of ligand molecules, suggesting the presence of a critical intermolecular distance for cells to effectively undergo oscillatory deformations. Fig. $6 \mathrm{~b}$ represents the calculated total power integrated over all modes as a function of $\left\langle d_{\mathrm{SDF} 1 \alpha}\right\rangle$ in order to highlight the impact of $\left\langle d_{\mathrm{SDF} 1 \alpha}\right\rangle$ on the total energy consumed by HSC in the presence (crosses) and absence (circles) of soluble SDF1 $\alpha$. In the absence of soluble SDF1 $\alpha$, the total power exhibited a distinct maximum at around $\left\langle d_{\mathrm{SDF} 1 a}\right\rangle=11$ and $18 \mathrm{~nm}$. Such a nonlinear behavior implied that HSC consumes the maximum energy by undergoing oscillatory motion and therefore, they are furthest from equilibrium at $\left\langle d_{\mathrm{SDF} 1 \alpha}\right\rangle=11$ and $18 \mathrm{~nm}$. Remarkably, this range agrees well with the characteristic average intermolecular distances for binding/unbinding transition determined by the pressure wave assay, $\left\langle d_{\mathrm{SDF} 1 \alpha}\right\rangle=14 \mathrm{~nm}$ (Fig. 4 ). In contrast, the total power exhibited no maximum over the whole range of $\left\langle d_{\mathrm{SDF} 1 \alpha}\right\rangle$ in the presence of $5 \mathrm{ng} / \mathrm{mL}$ soluble SDF1 $\alpha$ (Fig. 6b, red), suggesting that consumption of energy by HSC was significantly suppressed in the presence of soluble SDF1 $\alpha$ at the physiological level $(5 \mathrm{ng} / \mathrm{mL})$. It should be emphasized that the power spectrum analysis in Fourier space only enables one to discriminate the different modes $(m)$ of deformation and energy consumption of HSC in the presence and absence of chemo-attractant (SDF1 $\alpha$ ) at various $\left\langle d_{\mathrm{SDF} 1 \alpha}\right\rangle$, which cannot be assessed by the shape analysis in real space.

Similar to cells on membranes displaying SDF1 $\alpha$, we found that HSC on membranes displaying $\mathrm{N}$-cadherin predominantly undergo an oscillatory (mode $m=2$ ) deformation (Supplementary Fig. S6). In the absence of soluble SDF1 $\alpha$, the total power $\sum_{m} \hat{\Gamma}_{r, r}(\theta, t)$ of HSC on membranes displaying $\mathrm{N}$-cadherin is much less pronounced compared to those on SDF1 $\alpha$-functionalized membranes. The total power $\sum_{m} \hat{\Gamma}_{r, r}(\theta, t)$ was hardly influenced by $<d_{\mathrm{N}-\text { cadherin }}>$ or by
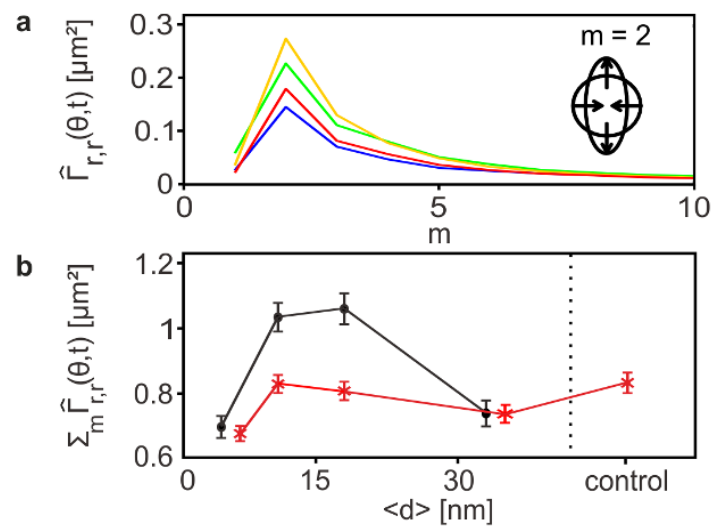

Figure 6 HSC locomotion is mostly based on oscillatory cell deformations. (a) Power spectrum of HSC at various $\left\langle d_{\mathrm{SDF} 1 \alpha}\right\rangle=6 \mathrm{~nm}$ (blue), $11 \mathrm{~nm}$ (green), $18 \mathrm{~nm}$ (orange) and $34 \mathrm{~nm}$ (red), indicating that mode $\mathrm{m}=2$ (inset) is dominant. (b) Total power in the presence (crosses) and absence (circles) of soluble SDF $1 \alpha$ plotted vs. $\left\langle d_{\text {SDF1 } \alpha}\right\rangle$. Data points represent means \pm SEM for $\mathrm{n}=30$ cells of three individual experiments.

the presence of soluble SDF1 $\alpha$. This finding can be attributed to the different role of $\mathrm{N}$-cadherin from SDF1 $\alpha$. The former serves as the cell adhesion molecule necessary for the anchorage of HSC to the bone marrow niche, while the latter functions as chemo-attractant for HSC migration. Thus, we concluded that the dynamic motion of HSC mediated by $\mathrm{N}$-cadherin is not directly influenced by soluble SDF1 $\alpha$.

Relevance for clinical stem cell transplantation. In the case of allogenic stem cell transplantations, HSC from a healthy donor are collected from peripheral blood (PB) after mobilization of HSC from $\mathrm{BM}$ with granulocyte colony-stimulating factor $(\mathrm{G}-\mathrm{CSF})^{43,44}$. It was shown that G-CSF induces the release of neutrophil elastase (NE) and matrix metalloproteinase-9 (MMP-9). This in turn downregulates levels of SDF1 $\alpha$ in BM by enzymatic degradation ${ }^{45}$, follwed by the cleavage of the N-terminus of CXCR $4^{46}$. Thus, the enforced mobilization of HSC led to a decisive distortion of the interaction between SDF1 $\alpha$-CXCR4. We therefore examined the adhesion behavior of HSC from PB to supported membranes functionalized with SDF1 $\alpha$ which might be considered as a model of homing of healthy HSC to the BM niche of the patients. Fig. 7 represents the adhesion behavior of HSC from $\mathrm{PB}$ as a function of $\left\langle d_{\mathrm{SDF} 1 \alpha}\right\rangle$, which was significantly different from that of HSC collected from CB. For example, the fraction of adhered HSC from $\mathrm{PB}$ at $\left\langle d_{\mathrm{SDF} 1 \alpha}\right\rangle=11 \mathrm{~nm}$ was $55 \%$, which was much less than that of HSC derived from CB (97\%). A similar observation was found for the average area of tight adhesion. This resulted in a shift of the unbinding transition to a critical distance $\left\langle d_{\mathrm{SDF} 1 \alpha^{*}}\right\rangle \sim 10 \mathrm{~nm}$ and a cooperativity coefficient $n \sim 1.3$, respectively (Supplementary Fig. S7). The critical pressure for cell detachment was $5.8 \mathrm{MPa}$ at $\left\langle d_{\mathrm{SDF} 1 \alpha}\right\rangle=11 \mathrm{~nm}$, which was $15-20 \%$ less than the corresponding value of $\mathrm{HSC}$ derived from $\mathrm{CB}, 7.0 \mathrm{MPa}$. We have subsequently examined if the in vitro niche model based on SDF1 $\alpha$-functionalized supported membranes could be utilized to identify differential adhesion of $\mathrm{CD}_{3} 4^{+}$leukemia cells versus normal HSC. In Fig. 7, the adhesion of PB leukemia blasts (LB) from an AML patient was compared with that of PB HSC from a healthy donor. The fraction of adherent LB (88\%) was about 1.5 times higher than that of HSC from healthy PB (55\%), while the difference from CB HSC (97\%) was much less pronounced.

\section{Discussion}

To quantify the adhesive mechanisms between human HSC and the $\mathrm{BM}$ niche, we have designed an in vitro surrogate surface model of 


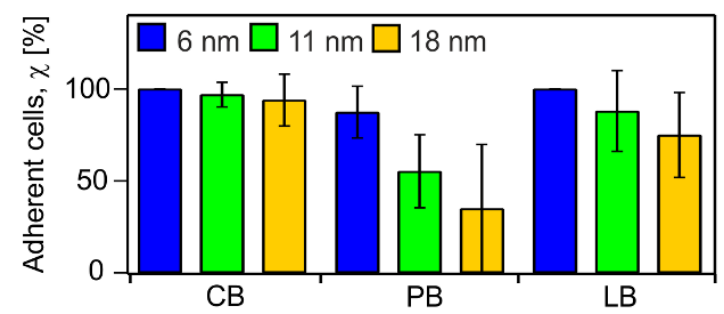

Figure 7 | Adhesion dynamics are significantly different between LB, CB and PB HSC. The fraction of adherent cells of HSC from cord blood (CB), HSC from peripheral blood (PB) and leukemia blast cells (LB) at $<d_{\mathrm{SDF} 1 \alpha}>\sim 6$ (blue), 11 (green), 18 (yellow), at $t=2 \mathrm{~h}$. Bars represent means \pm SD for $\mathrm{n}=50$ cells.

BM niche based on supported membranes displaying specific ligands $(\mathrm{N}$-cadherin and SDF1 $\alpha$ ) at defined intermolecular distances. This quantitative studies have enabled us to address several open questions in understanding the mechanisms with which the HSC bind to the niche.

First, HSC specifically adhered to the surrogate niche model via homophilic interactions mediated by $\mathrm{N}$-cadherin and heterophilic interactions mediated by SDF $1 \alpha$ and CXCR4. This was in stark contrast to the control experiments on pure phospholipid membranes (Fig. 2b), thus excluding the possibility of any non-specific adhesion. Second, the significant correlation between the surface density of adherent HSC and the average intermolecular distance between the corresponding ligand molecules $\langle d\rangle$ demonstrated that HSC sensitively detected minor changes in the intermolecular distance between $\mathrm{N}$-cadherin and SDF1 $\alpha^{7}$. Third, the area of tight adhesion calculated from RICM analysis permitted the more precise determination of the characteristic intermolecular distance for binding/ unbinding transition (Fig. 3B). Fourth, the laser-induced pressure waves enabled us to quantify the mechanical strength of the HSC adhesion on in vitro niche models (Fig. $4 \mathrm{~b}$ ), where the relative significance of two different molecular binding axes was determined in a quantitative manner.

Our results clearly demonstrate that HSC are able to bind to surrogate surfaces displaying $\mathrm{N}$-cadherin. This finding suggests their capability to interact with their niche via the homophilic N-cadherin axis. In fact, this is in good agreement with our previous study demonstrating that $\mathrm{N}$-cadherin is localized at the binding site between HSC and $\mathrm{MSC}^{7}$. The direct detection of $\mathrm{N}$-cadherin mediated adhesion of HSC is in stark contrast to other studies which failed to detect any role of $\mathrm{N}$-cadherin ${ }^{47-50}$. Moreover, we found that the adhesion strength mediated by heterophilic SDF1 $\alpha$-CXCR 4 axis was stronger than that mediated by homophilic $\mathrm{N}$-cadherin axis. We have also quantitatively determined the relevance of the counterplay between membrane-bound and soluble SDF $1 \alpha$ on the interaction between HSC and the surrogate niche model. As the mobilization/homing mechanism was regulated by a dynamic stimulation of the SDF1 $\alpha$ level in the BM, differences in the adhesion strength indicated that the BM niche was able to regulate homing versus mobilization by changing the ratio of soluble versus immobilized SDF1 $\alpha$ expressed by MSC.

The statistical analysis of morphological dynamics has demonstrated that HSC predominantly undergo oscillatory locomotion. The power spectra exhibited peaks at around $\langle d\rangle=11$ and $18 \mathrm{~nm}$ for SDF $1 \alpha$ and $\langle d\rangle=34 \mathrm{~nm}$ for $\mathrm{N}$-cadherin, respectively, corresponding to the unbinding transition points. This finding is intriguing, as the strongest shape deformation occurred at the binding/unbinding transition and did not scale with the average intermolecular distance between ligand molecules $\langle d\rangle$. The observed nonlinearity between HSC dynamics and $<d>$ implies that HSC was furthest from equilibrium around this $\langle d\rangle$, dissipating the maximum energy by undergoing oscillatory motion. The total power in the presence of soluble SDF1 $\alpha(5 \mathrm{ng} / \mathrm{mL})$ was always smaller than the corresponding values in its absence, suggesting that the energy dissipation by HSC is strongly damped in the presence of soluble SDF1 $\alpha$. It should be noted that all the previous in vitro studies relying merely on the phenomenological observation needed a concentration of soluble SDF1 $\alpha$ which was 1-2 orders of magnitude higher than ours in order to describe the change in cell morphology caused by SDF $1 \alpha^{36,37}$. This finding demonstrated that the analysis of morphological dynamics of live HSC in Fourier space (power spectrum calculation) is a very powerful tool to extract the non-equilibrium fluctuation dynamics of HSC, which is hidden behind the noise and thus cannot be detected by the conventional image analysis in real space. Our results implied that soluble SDF1 $\alpha$ not only interfered with the strength of adhesion mediated by CXCR4-SDF1 $\alpha$ but also affected the energy dissipation during the migration of HSC. The combination of quantitative force measurements and defined surrogate surfaces offers a unique advantage over previous in vitro adhesion assays on feeder MSC layers ${ }^{36,37}$, unraveling not only the relative significance of two key axis mediated by N-cadherin and CXCR4 but also their cross-talk with soluble chemoattractant SDF1 $\alpha$ in a quantitative manner. By extrapolating this approach we have compared the adhesion of HSC derived from PB mobilized by G-CSF and that of HSC from CB. The results suggested that GCSF treatment leads to a lower fraction of adherent cells and a decrease of the adhesion strength as compared to CB HSC. These findings supported the previous studies suggesting that G-CSF induced a release of NE and MMP-9, accompanied by a downregulation of SDF $1 \alpha$ in $\mathrm{BM}$ and the cleavage of the $\mathrm{N}$-terminus of CXCR4 by enzymatic degradation ${ }^{45}$ leading to a mobilization of HSC from $\mathrm{BM}$ to $\mathrm{PB}^{46}$. In contrast, the adhesive strength between $\mathrm{LB}$ and the niche might be higher compared to PB HSC. Albeit the number of samples tested was not yet adequate to address this important question, we have demonstrated that the combination of quantitative physical tools will enable us to define the differentiatial adhesion of normal HSC versus LSC to the marrow niche. The combination of precisely controlled in vitro niche models and new quantitative tools will provide us with significant information for the concept of mobilization of LSC towards the elimation of LSC for long-term remission.

\section{Methods}

Lipids, proteins. 1-stearoyl-2-oleoyl-sn-glycero-3-phosphocholine (SOPC), 1,2dioleoyl-sn-glycero-3-[(N-(5-amino-1-carboxypentyl)iminodiacetic acid)succinyl] (nickel salt) (DOGS-NTA $\left.\left(\mathrm{Ni}^{2+}\right)\right)$ and 1,2-dioleoyl-sn-glycero-phosphoethanolamine-3-N-(cap biotinyl) (biotin-DOPE) were purchased from Avanti Polar Lipids (Alabaster, USA), and neutravidin from Life Technologies. Recombinant stromal cell-derived factor- $1 \alpha(\mathrm{SDF} 1 \alpha)$ with and without biotin tags and human Ncadherin with histidine tag were purchased from Almac Group (Craigavon, UK) and R\&D Systems Inc. (Wiesbaden, Germany), respectively. For all cell experiments, Iscove's Modified Dulbecco's Media from Life Technologies was used.

Preparation of supported membranes. Glass slides were cleaned by a modified RCA protocol $^{51}$. Therefore, substrates were sonicated in acetone, ethanol, methanol and water for $3 \mathrm{~min}$, subsequently. Then, they were immersed in a solution of $1: 1: 5(\mathrm{v} / \mathrm{v} /$ v) $\mathrm{H}_{2} \mathrm{O}_{2}(30 \%) / \mathrm{NH}_{4} \mathrm{OH}(25 \%) / \mathrm{H}_{2} \mathrm{O}$ and sonicated at room temperature for $3 \mathrm{~min}$, before soaking them at $60^{\circ} \mathrm{C}$ for another $30 \mathrm{~min}$. Thereafter, substrates were rinsed intensively with ultrapure water, dried at $70^{\circ} \mathrm{C}$ and stored in a vacuum chamber at room temperature. Cell incubation chambers were prepared by sealing microscopic grade $256 \times 75 \mathrm{~mm}^{2}$ glass slides from Gerhard Menzel GmbH (Braunschweig, Germany) to bottomless plastic fluidic channels ( $\mu$-Slide $\mathrm{VI}^{0.4}$ ) from Ibidi (Martinsried, Germany) in case of RICM experiments. For picosecond laser pulse detachment assays, round microscopic grade glass slides with a diameter of $28 \mathrm{~mm}$ were bonded to bottomless culture dishes from Ibidi. Polydimethylsiloxane produced from base and curing agent (SYLGARD184, Dow Corning Co., USA) served as bonding agent for both chambers.

Stock solutions of lipids in $\mathrm{CHCl}_{3}(5 \mathrm{mg} / \mathrm{mL})$ were mixed to obtain the desired concentration of either DOGS-NTA $\left(\mathrm{Ni}^{2+}\right)$ or biotin-DOPE in SOPC. After evaporation of $\mathrm{CHCl}_{3}$ under a gentle nitrogen stream and storage under vacuum overnight, the lipids were re-suspended in HBS and sonicated with a titanium microtip sonicator S3000 (Misonix Inc., Farmingdale, USA) for $30 \mathrm{~min}$ to obtain small unilamellar vesicles (SUVs). To remove any residual titanium particles, vesicle suspensions were centrifuged (Eppendorf, Hamburg, Germany) for $10 \mathrm{~min}$ at $13400 \mathrm{~g}$. Thereafter, SUV suspensions $(1 \mathrm{mg} / \mathrm{mL})$ were stored at $4^{\circ} \mathrm{C}$. Solid-supported 
membranes were prepared by vesicle fusion ${ }^{19}$. Specifically, SUV suspensions were injected into the sealed cell incubation chamber and incubated for $30 \mathrm{~min}$ at $40^{\circ} \mathrm{C}$, followed by intensively rinsing with $\mathrm{HBS}$ buffer $(150 \mathrm{mM} \mathrm{NaCl}, 10 \mathrm{mM}$ Hepes, $\mathrm{pH}$ 7.5) to remove excess SUVs.

Functionalization of supported membranes N-cadherin and SDF1a. Supported membranes doped with DOGS-NTA $\left(\mathrm{Ni}^{2+}\right)$ were incubated with nickel buffer $(1 \mathrm{mM}$ $\mathrm{NiCl}_{2} \cdot 6 \mathrm{H}_{2} \mathrm{O}, 150 \mathrm{mM} \mathrm{NaCl}, 10 \mathrm{mM}$ Hepes, $\mathrm{pH}$ 7.5) for 45 min to saturate the nickel chelating nitrilotriacetic acid (NTA) headgroups. The buffer was exchanged to calcium buffer $\left(1 \mathrm{mM} \mathrm{CaCl}_{2} .2 \mathrm{H}_{2} \mathrm{O}, 150 \mathrm{mM} \mathrm{NaCl}, 10 \mathrm{mM}\right.$ Hepes, $\mathrm{pH}$ 7.5), then human recombinant His6 N-cadherin was added $(10 \mu \mathrm{g} / \mathrm{mL})$ and incubated for $12 \mathrm{~h}$ at room temperature. For the immobilization of SDF $1 \alpha$, supported membranes doped with biotin-DOPE were incubated with neutravidin solution $(40 \mu \mathrm{g} / \mathrm{mL})$ for $2 \mathrm{~h}$ at room temperature. After removing the neutravidin, biotinylated SDF1 $\alpha(10 \mu \mathrm{g} / \mathrm{mL})$ was added. Since the anchor lipids are monomerly incorporated into the matrix lipids, the average lateral distance between lipid anchors $\langle d\rangle$ and thus proteins can be estimated from the molar fraction $\mathrm{x}$ of lipid anchors by inserting the value of the lipid area of $A_{\text {lipid }} \sim 65 \AA^{252,53}$.

$$
\langle d\rangle=\sqrt{\frac{A_{\text {lipid }}}{x}}
$$

For both $\mathrm{N}$-cadherin and $\mathrm{SDF} 1 \alpha$, excess proteins were removed by rinsing extensively with cell culture medium prior to the cell adhesion experiments, and the samples were equilibrated at $37^{\circ} \mathrm{C}$. As we previously reported, the binding of histagged cadherins to NTA lipids and the neutravidin-biotin binding were confirmed using quartz-crystal microbalance with dissipation ${ }^{52}$ and grazing incidence $\mathrm{X}$-ray fluorescence ${ }^{54,55}$.

Isolation of human HSC. All of the experiments involving the use of human HSC were approved by the Ethics Committee of the Medical Faculty, University of Heidelberg, Germany and performed after obtaining informed consent from all voluntary donors in accordance with relevant guidelines and regulations. Human HSC, defined in this study as CD34 ${ }^{+}$cells, were derived from umbilical cord blood (CB) or from healthy allogeneic stem cell donors. The latter had received a mobilization regimen with G-CSF ( $10 \mu \mathrm{g} / \mathrm{kg}$ bw per day subcutaneously for 5 days) and a sample of $60 \mathrm{ml}$ of peripheral blood (PB) was taken for this study prior to leukapheresis. HSC were isolated as previously described ${ }^{7,56}$. Briefly, mononuclear cells (MNCs) were isolated by density gradient centrifugation using the FicollHypaque technique (Merck KGaA, Darmstadt, Germany). $\mathrm{CD}_{3} 4^{+}$cells from the MNC fraction were enriched by labelling with magnetic microbeads and sorted twice using an affinity column with the AutoMACS system (all Miltenyi Biotec GmbH, Bergisch-Gladbach, Germany). The cells were allowed to rest for at least 2 hours at $37^{\circ} \mathrm{C}$ and $5 \% \mathrm{CO}_{2}$ before further use and were stored in long-term bone marrow culture (LTBMC) medium, a basal HPC culture medium described by Dexter et al. ${ }^{57}$ consisting of 75\% Iscove's modified Dulbecco's medium (IMDM; Life Technologies Inc., Carlsbad, USA), 12.5\% FCS, 12.5\% horse serum (both Stemcell Technologies Inc., Vancouver, Canada), $2 \mathrm{mM} \mathrm{L}$-glutamine, $100 \mathrm{U} / \mathrm{ml}$ penicillin/streptomycin (Life Technologies) and 0.05\% hydrocortisone 100 (Sigma-Aldrich Co., St. Louis, USA). Staining with propidium iodide was performed to exclude non-viable cells. Reanalysis of the isolated cells by flow cytometry revealed a purity of $>95 \% \mathrm{CD} 34^{+}$ cells.

Isolation of leukemia blasts. All of the experiments involving the use of CD34 leukemia blasts (LB) were approved by the Ethics Committee of the Medical Faculty, University of Heidelberg, Germany and performed after obtaining informed consent from the voluntary donor in accordance with relevant guidelines and regulations. LB were collected from a patient with newly diagnosed acute myeloid leukemia (AML). LB from PB were isolated as described above for HSC from CB. After the isolation, the cells were stored in liquid nitrogen at $77 \mathrm{~K}$.

Cell adhesion experiments. HSC cell sample in LTBMC was separated, one portion was supplemented with soluble SDF1 $\alpha(5 \mathrm{ng} / \mathrm{mL})$ and both samples were preincubated for $2 \mathrm{~h}$ at $37^{\circ} \mathrm{C}$ and $5 \% \mathrm{CO}_{2}$. Before cell adhesion experiments, culture medium was exchanged to pre-warmed IMDM and cells were seeded at a density of 1 $\times 10^{5} \mathrm{cells} / \mathrm{cm}^{2}$ into cell incubation chambers in the absence and presence of soluble $\operatorname{SDF} 1 \alpha(5 \mathrm{ng} / \mathrm{mL})$. The viability of HSC was assessed before seeding and after $2 \mathrm{~h}$ and $4 \mathrm{~h}$ of seeding HSC on the in vitro niche model by performing a dye exclusion test using the diazo dye trypan blue. Additionally, cells were stained using Annexin V for apoptosis. In both cases and for all time points a cell viability of $\sim 90 \%$ was obtained, which did not change during the time period of the experiments (see Supplementary Fig. S8 and S9).

Reflection interference contrast microscopy. Non-invasive live cell imaging using reflection interference contrast microscopy (RICM) was performed on an Axiovert 200 inverted microscope (Carl Zeiss AG, Oberkochen, Germany) equipped with a PlanNeofluar 63x/1.25 Antiflex oil-immersion objective with a built-in lambdaquarter plate, a filter cube with crossed polarizers and a cell incubation chamber from Ibidi. To observe interference, light emerging from a metal halogenide lamp (X-Cite 120 PC, Lumen Dynamics Group Inc., Ontario, Canada) was filtered using a green filter $\left(\lambda_{\text {ex }}=546 \mathrm{~nm}\right)$ and passed perpendicular oriented polarizers before and after the first and second transmission across the lambda-quarter plate, respectively. Sequences of 20 consecutive images were acquired for at least 10 positions per condition using an Orca ER CCD camera (Hamamatsu Photonics, Hamamatsu, Japan) with an exposure time set to $0.1 \mathrm{~s}$ after $2 \mathrm{~h}$ of cell seeding. The aperture diaphragm was slightly opened (illumination numerical aperture (INA) $\sim 0.64$ ) in order to avoid multiple reflections within cells and loss of contrast. Image corrections for the parabolic illumination profile and shot noise were undertaken as described previously ${ }^{58}$. The measured intensity was converted into heights, applying the RICM theory for finite INA and multiple reflecting layers, with refractive indices $n_{1}=1.525$ (glass substrate), $n_{2}=1.486$ (lipid membrane), $n_{3}=1.335$ (IMDM medium) and $n=$ $1.37\left(\mathrm{cytosol}^{59}\right)$. The area of tight adhesion was calculated from the average height and standard deviation in each pixel.

Pressure wave assay. A Nd:YAG laser system $\left(\lambda=1064 \mathrm{~nm}, \tau_{\mathrm{L}}=60 \mathrm{ps}\right.$, PY 61C-20, Continuum, Santa Clara, CA, USA) was coupled to an inverted microscope (Eclipse TE2000-U, Nikon Europe) equipped with a self-built cell incubation chamber. Pressure waves were induced by focusing picosecond laser pulses over a PlanApo 20 $\times / 0.75$ objective into the sample chamber $(1.5 \mathrm{~mm}$ away from the region of interests, and $0.1 \mathrm{~mm}$ above the surface). To confirm the stability and statistical reliability of the assay, calibration measurements were performed before and after several series of experiments (see Supplementary Fig. 1). To determine the relationship between the pulse energy $E_{\text {pulse }}$ and the hydrodynamic pressure $P$ of the generated wave, a piezoelectric hydrophone was placed $1.5 \mathrm{~mm}$ away from the focal point of the ps laser pulse and $100 \mu \mathrm{m}$ above the substrate. Then, a data set was recorded displaying the hydrodynamic pressure $P$ as a function of the laser pulse energy $E_{\text {pulse }}$ prior to each cell experiment. Thereafter, the pressure affecting HSC can be deduced from the obtained calibration curve. Supplementary Figure S1 represents the hydrodynamic

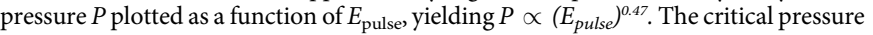
$P^{*}$ to detach $50 \%$ of adherent cells was defined as a new measure to quantify the binding strength. As the irradiation of an intensive, short laser pulse leads to the generation of a cavitation bubble at the position of the focal point, we carefully chose a measurement position at a distance where potential damage to HSC can be excluded. First, the distance between cells and the focal point of the laser $(1.5 \mathrm{~mm})$ was set to a value larger than the maximal possible radius of the cavitation bubble $\left(R_{\max }<\right.$ $1.4 \mathrm{~mm}$ ), which is at the same time larger than any damage observed on the in vitro niche model (with a maximal radial distance of $R_{\text {damage }}<300 \mu \mathrm{m}$ ). Second, the short separation distance between the focal point of the ps laser pulse and the substrate $(\sim 0.1 \mathrm{~mm})$ suppresses any adverse effects due to hydrodynamic liquid jet impacts. Thus, the target cells are not exposed to hot gaseous water ${ }^{60}$.

Time-lapse imaging. Movies of migrating cells were recorded with a Keyence BZ9000 (Keyence, Osaka, Japan) microscope, equipped with a Plan Fluor 40x/0.6 air objective. 1-2 positions per channel (and adhesion condition) were chosen and recording of phase contrast images was undertaken over 6 hours with a frame rate of $0,025 \mathrm{~Hz}$. Acquired movies were drift corrected utilizing cross-correlation analysis.

Statistics. The underlying number of experiments per data point corresponds to three experiments. In summary, the fraction of adhered cells $\chi$, the area of tight adhesion $A_{\text {Adh } h}$ and the critical detachment pressure $P^{*}$ were calculated for a total number of 50 cells per data point and data included in the corresponding figures represent means \pm standard deviation. Data characterising the morphological dynamics of HSC, comprise a total number of 30 cells per data point and represent means \pm standard deviation of the mean.

1. Zhang, J. et al. Identification of the haematopoietic stem cell niche and control of the niche size. Nature 425, 836-841 (2003).

2. Wagner, W. et al. Adhesion of hematopoietic progenitor cells to human mesenchymal stem cells as a model for cell-cell interaction. Exp. Hematol. 35 , 314-325 (2007).

3. Mendez-Ferrer, S. et al. Mesenchymal and haematopoietic stem cells form a unique bone marrow niche. Nature 466, 829-834 (2010).

4. Punzel, M. et al. The symmetry of initial divisions of human hematopoietic progenitors is altered only by the cellular microenvironment. Exp. Hematol. 31, 339-347 (2003)

5. Yamaguchi, M. et al. Ex vivo expansion of human UC blood primitive hematopoietic progenitors and transplantable stem cells using human primary BM stromal cells and human AB serum. Cytotherapy 4, 109-118 (2002).

6. Calvi, L. M. et al. Osteoblastic cells regulate the haematopoietic stem cell niche. Nature 425, 841-846 (2003).

7. Wein, F. et al. N-Cadherin is expressed on human hematopoietic progenitor cells and mediates interaction with human mesenchymal stromal cells. Stem Cell Res. 4, 129-139 (2010)

8. Puch, S. et al. N-cadherin is developmentally regulated and functionally involved in early hematopoietic cell differentiation. J. Cell Sci. 114, 1567-1577 (2001).

9. Hosokawa, K. et al. Cadherin-based adhesion is a potential target for niche manipulation to protect hematopoietic stem cells in adult bone marrow. Cell Stem Cell 6, 194-198 (2010).

10. Ponomaryov, T. et al. Induction of the chemokine stromal-derived factor-1 following DNA damage improves human stem cell function. J. Clin. Invest. 106, 1331-1339 (2000). 
11. Mohle, R. et al. The chemokine receptor CXCR-4 is expressed on CD34(+) hematopoietic progenitors and leukemic cells and mediates transendothelial migration induced by stromal cell-derived factor-1. Blood 91, 4523-4530 (1998).

12. Aiuti, A., Webb, I. J., Bleul, C., Springer, T. \& GutierrezRamos, J. C. The chemokine SDF-1 is a chemoattractant for human CD34(+) hematopoietic progenitor cells and provides a new mechanism to explain the mobilization of CD34(+) progenitors to peripheral blood. J. Exp. Med. 185, 111-120 (1997)

13. Peled, A. et al. The chemokine SDF-1 stimulates integrin-mediated arrest of CD34 + cells on vascular endothelium under shear flow. J. Clin. Invest. 104, 1199-1211 (1999).

14. Lowenberg, B., Downing, J. R. \& Burnett, A. Acute Myeloid Leukemia. New Engl. J. Med. 341, 1051-1062 (1999).

15. McCulloch, E. Stem cells in normal and leukemic hemopoiesis (Henry Stratton Lecture, 1982). Blood 62, 1-13 (1983).

16. Bonnet, D. \& Dick, J. E. Human acute myeloid leukemia is organized as a hierarchy that originates from a primitive hematopoietic cell. Nat. Med. 3 , 730-737 (1997).

17. Ishikawa, F. et al. Chemotherapy-resistant human AML stem cells home to and engraft within the bone-marrow endosteal region. Nat. Biotechnol. 25, 1315-1321 (2007)

18. Lane, S. W., Scadden, D. T., Gilliland, D. G. The leukemic stem cell niche: current concepts and therapeutic opportunities. Blood 114, 1150-1157 (2009).

19. Sackmann, E. Supported membranes: scientific and practical applications. Science 271, 43-48 (1996).

20. Tanaka, M. \& Sackmann, E. Polymer-supported membranes as models of the cell surface. Nature 437, 656-663 (2005).

21. Rädler, J. \& Sackmann, E. Imaging optical thicknesses and separation distances of phospholipid vesicles at solid surfaces. J. Phys. II France 3, 727-748 (1993).

22. Yoshikawa, H. Y. et al. Quantitative Evaluation of Mechanosensing of Cells on Dynamically Tunable Hydrogels. J. Am. Chem. Soc. 133, 1367-1374 (2011).

23. Finer, J. T., Simmons, R. M. \& Spudich, J. A. Single myosin molecule mechanics: piconewton forces and nanometre steps. Nature 368, 113-119 (1994).

24. Bausch, A. R., Möller, W. \& Sackmann, E. Measurement of Local Viscoelasticity and Forces in Living Cells by Magnetic Tweezers. Biophys. J. 76, 573-579 (1999).

25. Yamamoto, A., Mishima, S., Maruyama, N. \& Sumita, M. A new technique for direct measurement of the shear force necessary to detach a cell from a material. Biomaterials 19, 871-879 (1998).

26. Maeda, Y. T., Inose, J., Matsuo, M. Y., Iwaya, S. \& Sano, M. Ordered Patterns of Cell Shape and Orientational Correlation during Spontaneous Cell Migration. PLoS One 3, e3734 (2008).

27. Kaindl, T. et al. Spatio-Temporal Patterns of Pancreatic Cancer Cells Expressing CD44 Isoforms on Supported Membranes Displaying Hyaluronic Acid Oligomers Arrays. PLoS One 7, e42991 (2012).

28. Barcroft, J. \& Hill, A. V. The nature of oxyhaemoglobin, with a note on its molecular weight. J. Physiol. 39, 411-428 (1910).

29. Walenda, T. et al. Co-culture with mesenchymal stromal cells increases proliferation and maintenance of haematopoietic progenitor cells. J. Cell. Mol. Med. 14, 337-350 (2010)

30. Merkel, R., Nassoy, P., Leung, A., Ritchie, K. \& Evans, E. Energy landscapes of receptor-ligand bonds explored with dynamic force spectroscopy. Nature 397, 50-53 (1999).

31. Francis, G. W., Fisher, L. R., Gamble, R. A. \& Gingell, D. Direct measurement of cell detachment force on single cells using a new electromechanical method. J. Cell Sci. 87, 519-523 (1987)

32. Pierrat, S., Brochard-Wyart, F. \& Nassoy, P. Enforced Detachment of Red Blood Cells Adhering to Surfaces: Statics and Dynamics. Biophys. J. 87, 2855-2869 (2004)

33. Gether, U. Uncovering Molecular Mechanisms Involved in Activation of G Protein-Coupled Receptors. Endocr. Rev. 21, 90-113 (2000).

34. Leckband, D. E., le Duc, Q., Wang, N. \& de Rooij, J. Mechanotransduction at cadherin-mediated adhesions. Curr. Opin. Cell Biol. 23, 523-530 (2011).

35. Faber, A. et al. The many facets of SDF-1alpha, CXCR4 agonists and antagonists on hematopoietic progenitor cells. J. Biomed. Biotechnol. 2007, 26065 (2007)

36. Voermans, C., Gerritsen, W. R., von dem Borne, A. E. G. K. \& van der Schoot, C. E. Increased migration of cord blood-derived CD34+ cells, as compared to bone marrow and mobilized peripheral blood CD34+ cells across uncoated or fibronectin-coated filters. Exp. Hematol. 27, 1806-1814 (1999).

37. Fruehauf, S., Srbic, K., Seggewiss, R., Topaly, J. \& Ho, A. D. Functional characterization of podia formation in normal and malignant hematopoietic cells. J. Leukocyte Biol. 71, 425-432 (2002).

38. Brochard, F. \& Lennon, J. Frequency spectrum of the flicker phenomenon in erythrocytes. J. Phys. (Paris) 36, 1035-1047 (1975).

39. Gov, N. S. \& Safran, S. A. Red Blood Cell Membrane Fluctuations and Shape Controlled by ATP-Induced Cytoskeletal Defects. Biophys. J. 88, 1859-1874 (2005).

40. Betz, T., Lenz, M., Joanny, J.-F. \& Sykes, C. ATP-dependent mechanics of red blood cells. Proc. Natl. Acad. Sci. U. S. A. 106, 15320-15325 (2009).

41. Cell mechanics (eds Wang, Y.-L., Discher, D. E.). Academic Press (2007).

42. Partin, A. W., Schoeniger, J. S., Mohler, J. L. \& Coffey, D. S. Fourier analysis of cell motility: correlation of motility with metastatic potential. Proc. Natl. Acad. Sci. U. S. A. 86, 1254-1258 (1989)

43. Russell, N. H., Hunter, A., Rogers, S., Hanley, J. \& Anderson, D. Peripheral blood stem cells as an alternative to marrow for allogeneic transplantation. Lancet 341, 1482 (1993)
44. Kessinger, A. et al. Allogeneic transplantation of blood-derived, T cell-depleted hemopoietic stem cells after myeloablative treatment in a patient with acute lymphoblastic leukemia. Bone Marrow Transplant. 4, 643-646 (1989).

45. Petit, I. et al. G-CSF induces stem cell mobilization by decreasing bone marrow SDF-1 and up-regulating CXCR4. Nat. Immunol. 3, 687-694 (2002)

46. Lévesque, J.-P., Hendy, J., Takamatsu, Y., Simmons, P. J. \& Bendall, L. J. Disruption of the CXCR4/CXCL12 chemotactic interaction during hematopoietic stem cell mobilization induced by GCSF or cyclophosphamide. J. Clin. Invest. 111, 187-196 (2003).

47. Bromberg, O. et al. Osteoblastic N-cadherin is not required for microenvironmental support and regulation of hematopoietic stem and progenitor cells. Blood 120, 303-313 (2012).

48. Greenbaum, A. M., Revollo, L. D., Woloszynek, J. R., Civitelli, R. \& Link, D. C. Ncadherin in osteolineage cells is not required for maintenance of hematopoietic stem cells. Blood 120, 295-302 (2012).

49. Kiel, M. J., Acar, M., Radice, G. L. \& Morrison, S. J. Hematopoietic stem cells do not depend on N-cadherin to regulate their maintenance. Cell Stem Cell 4, 170-179 (2009).

50. Li, P. \& Zon, L. I. Resolving the Controversy about N-Cadherin and Hematopoietic Stem Cells. Cell Stem Cell 6, 199-202 (2010).

51. Kern, W. \& Puotinen, D. A. Cleaning solutions based on hydrogen peroxide for use in silicon semiconductor technology. RCA Rev. 31, 187-206 (1970).

52. Körner, A. et al. Cell Differentiation of Pluripotent Tissue Sheets Immobilized on Supported Membranes Displaying Cadherin-11. PLoS One 8, e54749 (2013).

53. Sackmann, E. \& Lipowsky, R. Structure and dynamics of membranes (eds). Elsevier Science (1995).

54. Abuillan, W. et al. Quantitative determination of the lateral density and intermolecular correlation between proteins anchored on the membrane surfaces using grazing incidence small-angle X-ray scattering and grazing incidence X-ray fluorescence. J. Chem. Phys. 137, 204907-204908 (2012).

55. Körner, A. et al. Quantitative Determination of Lateral Concentration and Depth Profile of Histidine-Tagged Recombinant Proteins Probed by Grazing Incidence X-ray Fluorescence. J. Phys. Chem. B 117, 5002-5008 (2013).

56. Ludwig, A. et al. Functional potentials of human hematopoietic progenitor cells are maintained by mesenchymal stromal cells and not impaired by plerixafor. Cytotherapy 16, 111-121 (2014).

57. Dexter, T. M. \& Moore, M. A. S. In vitro duplication and cure of haemopoietic defects in genetically anaemic mice. Nature 269, 412-414 (1977).

58. Monzel, C., Fenz, S. F., Merkel, R. \& Sengupta, K. Probing Biomembrane Dynamics by Dual-Wavelength Reflection Interference Contrast Microscopy. Chemphyschem 10, 2828-2838 (2009).

59. Coelho Neto, J., Agero, U., Gazzinelli, R. T. \& Mesquita, O. N. Measuring optical and mechanical properties of a living cell with defocusing microscopy. Biophys. J. 91, 1108-1115 (2006).

60. Vogel, A., Lauterborn, W. \& Timm, R. Optical and acoustic investigations of the dynamics of laser-produced cavitation bubbles near a solid boundary. J. Fluid Mech. 206, 299-338 (1989).

\section{Acknowledgments}

The authors thank the German Research Foundation (Collaborative Research Center, SFB 873 B 07) and A. Lenze for technical supports. A.D.H. is thankful to the Thiele-Stiftung for financial support, P.W. and A.D.H. to the German Ministry of Education and Research ("Systems Biology of Erythropoietin", No. 0316182d), and M.T. to Sumitomo Foundation, EU FP7 "SoftActive", MEXT (No. 26103521), and JSPS (No. 26247070). M.T. is a member of German Excellence Cluster "CellNetwork" and Helmholtz Program "BioInterface”. C.M. thanks "CellNetwork" for the postdoctoral fellowship. A.S.B. thanks the Baden Württemberg State Foundation for the fellowship and Helmholtz BioInterfaces International Graduate School. H.Y.Y. thanks JSPS for KAKENHI (Nos. 24680050 and 24106505) and the Brain Circulation Program. iCeMS is supported by World Premier International Research Center Initiative (WPI), MEXT (Japan).

\section{Additional information}

Author contributions M.T. and A.D.H. designed the research, A.S.B., C.M., H.Y.Y., P.W., V.E. and R.S. performed research, and A.S.B., C.M., M.T. and A.D.H. wrote the paper.

Supplementary information accompanies this paper at http://www.nature.com/ scientificreports

Competing financial interests: The authors declare no competing financial interests.

How to cite this article: Burk, A.S. et al. Quantifying Adhesion Mechanisms and Dynamics of Human Hematopoietic Stem and Progenitor Cells. Sci. Rep. 5, 9370; DOI:10.1038/ srep09370 (2015)

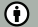

This work is licensed under a Creative Commons Attribution 4.0 International License. The images or other third party material in this article are included in the article's Creative Commons license, unless indicated otherwise in the credit line; if the material is not included under the Creative Commons license, users will need to obtain permission from the license holder in order to reproduce the material. To view a copy of this license, visit http://creativecommons.org/licenses/by/4.0/ 